\title{
Antitrust Guidelines: A Simple Operational Method for Evaluating Horizontal Mergers
}

Davide Dragone, Luca Lambertini and Andrea Mantovani Department of Economics, University of Bologna

Strada Maggiore 45, 40125 Bologna, Italy

phone: +39-051-2092600; fax: +39-051-2092664

dragone@spbo.unibo.it; lamberti@spbo.unibo.it; mantovan@spbo.unibo.it

March 20, 2007 


\begin{abstract}
We reexamine the profitability and social efficiency of horizontal mergers in a Cournot oligopoly with decreasing average costs. Assuming the merger allows for efficiency gains in production, we identify the conditions under which the merger is, respectively, profitable and socially desirable. The economic preditions of the model are contrasted with FTC guidelines, based on a simple method that allows to forecast the economic consequances of a merger in terms of ex ante observables. This comparative assessment highlights the existence of well defined parameter regions where FTC guidelines lead to systematic errors.
\end{abstract}

JEL Classification: D43, G34, L13

Keywords: horizontal merger, oligopoly, efficiency 


\section{Introduction}

Antitrust regulation on horizontal mergers is based on the use of the HerfindahlHirschman index (HHI). This index is easy to use as it only needs information on market shares. Moreover, it is very appealing because it relies on the idea that concentrated markets are harmful for consumers. However, this approach neglects potential efficiency gains resulting from adjustments in fixed costs via the merger. If social desirability of the merger is to be evaluated considering both consumer surplus and overall industry profits, i.e. by the conventional utilitarian social welfare function, then the $\mathrm{HH}$ index can be misleading, as already observed by Farrell and Shapiro (1990, 2000). Nonetheless, while the economic foundation of their critics is sound, they did not provide an alternative tool for the Antitrust authority.

The aim of this paper is precisely to propose an alternative measure for evaluating horizontal mergers. In particular, we consider a standard social welfare argument and propose a tool that can be managed also by non-practitioners, as it is expressed only by observable variables such as the number of firms, production's outcome and costs.

We start from a situation where a synergy operates, in such a way that the overall fixed cost associated with the merger is a fraction of the sum of the fixed costs borne ex ante by the firms taking part into the merger. We compare ex ante and ex post social welfare in such a way that only measurable variables appear and discover when the proposed merger is socially and privately desirable. We point out the minimal requirement in terms of efficiency gains required to allow the proposed merger to take place. More precisely, we find the following results: first, if the fixed cost savings generated by the 
merger are large enough, then the merger becomes profitable irrespective of the number of firms involved. Likewise, there exists a critical threshold of such efficiency gain above which the merger is socially desirable. The ranking of the two thresholds depend on the number of firms in the industry before the merger takes place. If the size of the merger (that is, the number of firms involved in it) is small enough, then we observe the counterintuitive and, thus far, neglected situation where the merger is socially convenient while being not profitable. If this is the case, then the policy maker may induce firms to merge by subsidising them (for instance, by using funds raised through taxes) provided that the representative shareholder is sufficiently atomistic to ensure that future dividends compensate current taxation.

After exposing the theoretical framework, we proceed to compare its normative implications against the current merger guidelines adopted by the Federal Trade Commission. These are based on the Herfindal-Hirschman index, which of course is an indirect indicator, rather than the demand and cost parameters belonging to the economic model. Therefore, in order to carry out the comparative assessment, we design a simple method based upon ex ante observable magnitudes only. This allows us to identify the occurrence of errors in both directions, i.e., to allow a merger that should be forbidden and conversely, produced by the application of FTC guidelines. Moreover, we also identify qualitative conditions under which a merger to monopoly is both privately and socially convenient. As a last step, we provide some extensions of our method to different setups, allowing for (i) asymmetric marginal costs across firms, (ii) increasing marginal costs, and (iii) non-linear market demand. 
The remainder of the paper is laid out as follows. The model is outlined in section 2. The assessment of the effectiveness of FTC guidelines in the light of the theoretical predictions is carried out in section 3. Extensions are in section 4 . Section 5 concludes.

\section{The model}

We examine a Cournot oligopoly where, ex ante, $n$ firms sell a homogeneous good whose demand function is $p=a-\sum_{i=1}^{n} q_{i}$. Each firm uses a technology characterised by increasing returns to scale, summarised by the cost function $C_{i}=c q_{i}+F$, where $c>0$ is the marginal cost and $F>0$ is a fixed cost.

We first consider a market where $n$ firms compete simultaneously. Therefore, individual outputs and profits at the symmetric Nash equilibrium are:

$$
\begin{aligned}
q_{e a} & =\frac{a-c}{n+1} ; \\
\pi_{e a} & =\frac{(a-c)^{2}}{(n+1)^{2}}-F
\end{aligned}
$$

where the subscript ea indicates ex ante values. The corresponding consumer surplus is:

$$
C S_{e a}=\frac{n^{2}(a-c)^{2}}{2(n+1)^{2}} .
$$

Hence, social welfare is $S W_{e a}=n \pi_{e a}+C S_{e a}$. For future reference, we express the previous expressions as a function of individual quantities:

$$
\begin{aligned}
\pi_{e a} & =q_{e a}^{2}-F \\
C S_{e a} & =\frac{n^{2}}{2} q_{e a}^{2} \\
S W_{e a} & =n\left(1+\frac{n}{2}\right) q_{e a}^{2}-F
\end{aligned}
$$


Before examining the feasibility of any horizontal merger, it must be true that the $n$ independent firms do survive at the initial Cournot Nash equilibrium. This requires $F<F_{e a} \triangleq q_{e a}^{2}$.

Now consider the situation where $m \in[2, n-1]$ firms evaluate the perspective of merging horizontally. We suppose that the merger gives rise to a reorganization of production plants within the resulting firm; in particular, we assume that the amount of fixed cost be equal to $\widehat{F} \triangleq(1+b) F$, where $b \in[0, m-1]$ is an inverse measure of the efficiency gains generated by the merger. ${ }^{1}$ Therefore, for all $b<m-1$, the usual argument whereby a merger is justified on efficiency grounds applies in the form of a reduction of the total amount of fixed cost borne by society.

When the merger is carried out, $n-m+1$ firms remain on the market; outputs, profits and consumer surplus modify as follows:

$$
\begin{aligned}
q_{e p} & =\frac{a-c}{n-m+2} \\
\widehat{\pi}_{e p} & =\frac{(a-c)^{2}}{(n-m+2)^{2}}-(1+b) F \\
\tilde{\pi}_{e p} & =\frac{(a-c)^{2}}{(n-m+2)^{2}}-F \\
C S_{e p} & =\frac{(n-m+1)^{2}(a-c)^{2}}{2(n-m+2)^{2}}
\end{aligned}
$$

where $\widehat{\pi}_{e p}$ is the profit of the firm resulting from the merger while $\tilde{\pi}_{e p}$ is the profit accruing to each of the $n-m$ firms that have remained independent. The associated social welfare is

$$
S W_{e p}=\widehat{\pi}_{e p}+(n-m) \tilde{\pi}_{e p}+C S_{e p} .
$$

\footnotetext{
${ }^{1}$ If $b=m-1$, then we are back to the case considered by Farrell and Shapiro (1990).
} 
In order to assess the private and social desirability of any given merger, one should compare ex post and ex ante levels of (insider) profits and social welfare. However, what usually prevents antitrust agencies from using the predictions of the theoretical model in such a straightforward way is the fact that market size $a-c$ is not known (or its measurement would take too long and far too much money). Accordingly, guidelines usually prescribe simple rules defined in terms of market shares and concentration indices. Yet, if one dwells upon the fact that ex ante output levels are indeed available from data bases, this obstacle may well become immaterial. To see this, note that the ex ante and ex post magnitudes of profits and social welfare can be easily redefined in terms of ex ante output levels only. In line with what is implicitly assumed when using concentration indices, similarly we assume that both the reservation price and the marginal cost of production are not altered when the merger takes place. This assumption appears as a reasonable one, since the merger has no impact on market size. Provided $(a-c)$ remains constant, from (1) we have:

$$
a-c=(n+1) q_{e a}
$$

We can therefore write all ex post expressions as a function of the ex ante 
quantity as follows:

$$
\begin{aligned}
q_{e p} & =\frac{n+1}{n-m+2} q_{e a} ; \\
\widehat{\pi}_{e p} & =\frac{(n+1)^{2}}{(n-m+2)^{2}} q_{e a}^{2}-(1+b) F \\
\tilde{\pi}_{e p} & =\frac{(n+1)^{2}}{(n-m+2)^{2}} q_{e a}^{2}-F ; \\
C S_{e p} & =\frac{(n-m+1)^{2}(n+1)^{2}}{2(n-m+2)^{2}} q_{e a}^{2} ; \\
S W_{e p} & =\frac{(n+1)^{2}[(m-n-3)(m-n-1)]}{2(n-m+2)^{2}} q_{e a}^{2}-(n-m+b+1) F .
\end{aligned}
$$

The advantage of this notation is that all ex post outcomes are easily ascertained by simply considering ex ante variables, such as individual quantities, total number of firms, potential candidates to the merger, fixed cost of production and the efficiency gain (which is assumed to be perfectly observable).

Given that any merger modifies market structure but also the distribution of fixed costs across firms in the industry, before proceeding to evaluate the private and social desirability of a merger we must ensure that firms are indeed viable at the corresponding ex post equilibrium. This requires the condition $\widehat{\pi}_{e p}>0 \Leftrightarrow F<F_{e p} \triangleq(n+1)^{2} q_{e a}^{2} /\left[(n-m+2)^{2}(1+b)\right] .^{2}$

On the basis of the previous analysis, we can evaluate the social and private desirability of the merger by comparing ex post and ex ante welfare and profits. This allows to state the following proposition:

Proposition 1 For all admissible levels of $\{b, n, m\}$, there exist the thresh-

\footnotetext{
${ }^{2}$ Note that this suffices to ensures that also $\tilde{\pi}_{e p}>0$, i.e., the outsiders' ex post profits be positive.
} 
old values of fixed costs $F_{\pi}$ and $F_{S W}$ above which the merger involving $m$ firms is, respectively, privately and socially desirable. If $n=3$ and $m=2$, then $F_{\pi}<F_{S W}$. If instead $n \geq 4$, then (i) $F_{\pi}>F_{S W}$ if $m$ is sufficiently small, and (ii) $F_{\pi}<F_{S W}$ if $m$ is sufficiently large.

Proof. The private (profit) incentive to carry out the merger requires:

$$
\begin{gathered}
\frac{\widehat{\pi}_{e p}}{m}>\pi_{e a} \Leftrightarrow \\
F>F_{\pi} \triangleq \frac{\left[m(n-m+2)^{2}-(n+1)^{2}\right]}{(m-b-1)(n-m+2)^{2}} q_{e a}^{2}
\end{gathered}
$$

while the social incentive requires:

$$
\begin{gathered}
S W_{e p}>S W_{e a} \Leftrightarrow \\
F>F_{S W} \triangleq \frac{(m-1)(2 n-m+3)}{2(m-1-b)(n-m+2)^{2}} q_{e a}^{2} .
\end{gathered}
$$

Comparing $F_{\pi}$ and $F_{S W}$, we obtain $F_{\pi}=F_{S W}$ at

$$
m_{1}=\frac{5}{4}+n-\frac{1}{4} \sqrt{24 n+33} ; m_{2}=\frac{5}{4}+n+\frac{1}{4} \sqrt{24 n+33} .
$$

Clearly, $m_{2}>n$ and can be disregarded; $m_{1}$ is always smaller than $n$, but $m_{1} \geq 2$ if and only if $n \geq 4$. If $n=3$, we have a special case where $m_{1}=1.688$. Therefore, we have two cases:

(i) for $n=3$ and $m=2$

$$
F_{\pi}=\frac{(n+1)^{2}}{72(1-b)} q_{e a}^{2}<F_{S W}=\frac{7(n+1)^{2}}{288(1-b)} q_{e a}^{2} ;
$$

(ii) for all $n \geq 4$,

$$
\begin{gathered}
F_{\pi}>F_{S W} \text { for all } m \in\left[2, m_{1}\right) ; \\
F_{\pi}<F_{S W} \text { for all } m \in\left(m_{1}, n-1\right] .
\end{gathered}
$$


This concludes the proof.

Now take the special case where $n=3$ and $m=2$. Here, given that the a priori degree of concentration of the industry is very high, the critical threshold for $F$ above which the antitrust agency permits the merger is necessarily higher than that above which firms find it profitable.

The perspective changes significantly for all $n \geq 4$. Provided that, as $n$ increases, the market becomes progressively more competitive, then $m_{1}$ becomes relevant and determines the presence of an interval where relatively small mergers are socially desirable. Note that $m_{1}$ is monotonically increasing in $n$. To this regard, it is worth stressing that, for all $F \in\left(F_{S W}, F_{\pi}\right)$, any merger involving $m \in\left[2, m_{1}\right)$ is socially convenient while it is not privately so. That is, we have the seemingly counterintuitive result whereby the antitrust agency would like the $m$ firms to merge, whereas they prefer to remain independent. To the best of our knowledge, this possibility has been overlooked so far in the existing literature. The reason of this result is that $m$ is small. This entails two related consequences: the first is that, for any given $b$, the reduction in fixed costs enjoyed by the firms involved in the merger is limited; the second is that the reduction in the overall number of firms in the industry after the merger has taken place is also limited. The first fact makes the merger unattractive to firms, while the second makes it appealing for the regulator. When this is the case, the policy maker may design an income transfer from consumers to the merging firms by means of a lump sum tax that becomes a subsidy to firms, so as to make the merger attractive to them. The total subsidy amounts to

$$
\left(\pi_{e a}-\frac{\widehat{\pi}_{e p}}{m}+\varepsilon\right) m
$$


where $\varepsilon$ is positive and arbitrarily small. This of course has no redistributive effects provided that all agents holds symmetric shares of the stock of those firms that are to carry out the merger.

\section{The economic model vs FTC guidelines}

Consider the 1992 FTC Horizontal Mergers Guidelines for evaluating prospect mergers. The general standards are based on the Herfindahl-Hirschman Index ("HHI") of market concentration, which is calculated by summing the squares of the individual market shares (in percentage terms) of all the participants as follows:

a) Post-Merger HHI Below 1000 [...] Mergers resulting in unconcentrated markets are unlikely to have adverse competitive effects and ordinarily require no further analysis.

b) Post-Merger HHI Between 1000 and 1800 [...] Mergers producing an increase in the HHI of less than 100 points in moderately concentrated markets post-merger are unlikely to have adverse competitive consequences and ordinarily require no further analysis. Mergers producing an increase in the HHI of more than 100 points in moderately concentrated markets post-merger potentially raise significant competitive concerns $[. .$.$] .$

c) Post-Merger HHI Above 1800. [...] Mergers producing an increase in the HHI of less than 50 points, even in highly concentrated markets post-merger, are unlikely to have adverse competitive consequences and ordinarily require no further analysis. 


\begin{abstract}
Mergers producing an increase in the HHI of more than 50 points in highly concentrated markets post-merger potentially raise significant competitive concerns [...] Where the post-merger HHI exceeds 1800, it will be presumed that mergers producing an increase in the HHI of more than 100 points are likely to create or enhance market power or facilitate its exercise. [...]
\end{abstract}

(1992 HORIZONTAL

\title{
MERGER GUIDELINES)
}

In view of the above FTC rules, we proceed now to evaluate the indications emerged from our model in order to obtain simple rules apt to evaluate the admissibility of horizontal mergers, in such a way that they can be easily compared with the FTC guidelines.

First of all, we focus on the space $\{n, m\}$. In Figure 1, the private and social efficiency of horizontal mergers (given by conditions (13-14)) are represented by the two curves, and we consider the case in which the subspace where $S W_{e p}-S W_{e a} \triangleq \Delta S W \geq 0$ is a proper subset of the subspace where $\widehat{\pi}_{e p} / m-\pi_{e a} \triangleq \Delta \pi>0$ for any triple $\left(b, F, q_{e a}\right)$. Provided that a merger proposal is submitted by firms to the antitrust authority, we can take for granted that $\Delta \pi>0$ holds. Accordingly, we confine our attention to the region $\Delta S W \geq 0,{ }^{3}$ to observe that the border of this region, i.e., the curve $\Delta S W=0$, may (i) lie always below and the right of the line $m=n$; (ii) be tangent to $m=n$; or (iii) intersect $m=n$ twice. The locus $\Delta S W=0$ shifts leftwards when either $F$ grows larger or $b$ decreases (intuitively, in both cases the efficiency gain produced by the merger is larger and therefore more

\footnotetext{
${ }^{3}$ This amounts to assuming that $F \in\left[F_{S W}, \min \left\{F_{e a}, F_{e p}\right\}\right)$.
} 
easily justifies the merger itself). The interesting implication of this fact is that whenever $m=n$ and $\Delta S W=0$ intersect (Figure 2) or are tangent to each other (Figure 3), then a merger to monopoly would be socially desirable in that it allows so high an efficiency gain that natural monopoly is the most desirable industry structure.

\section{Figure 1}

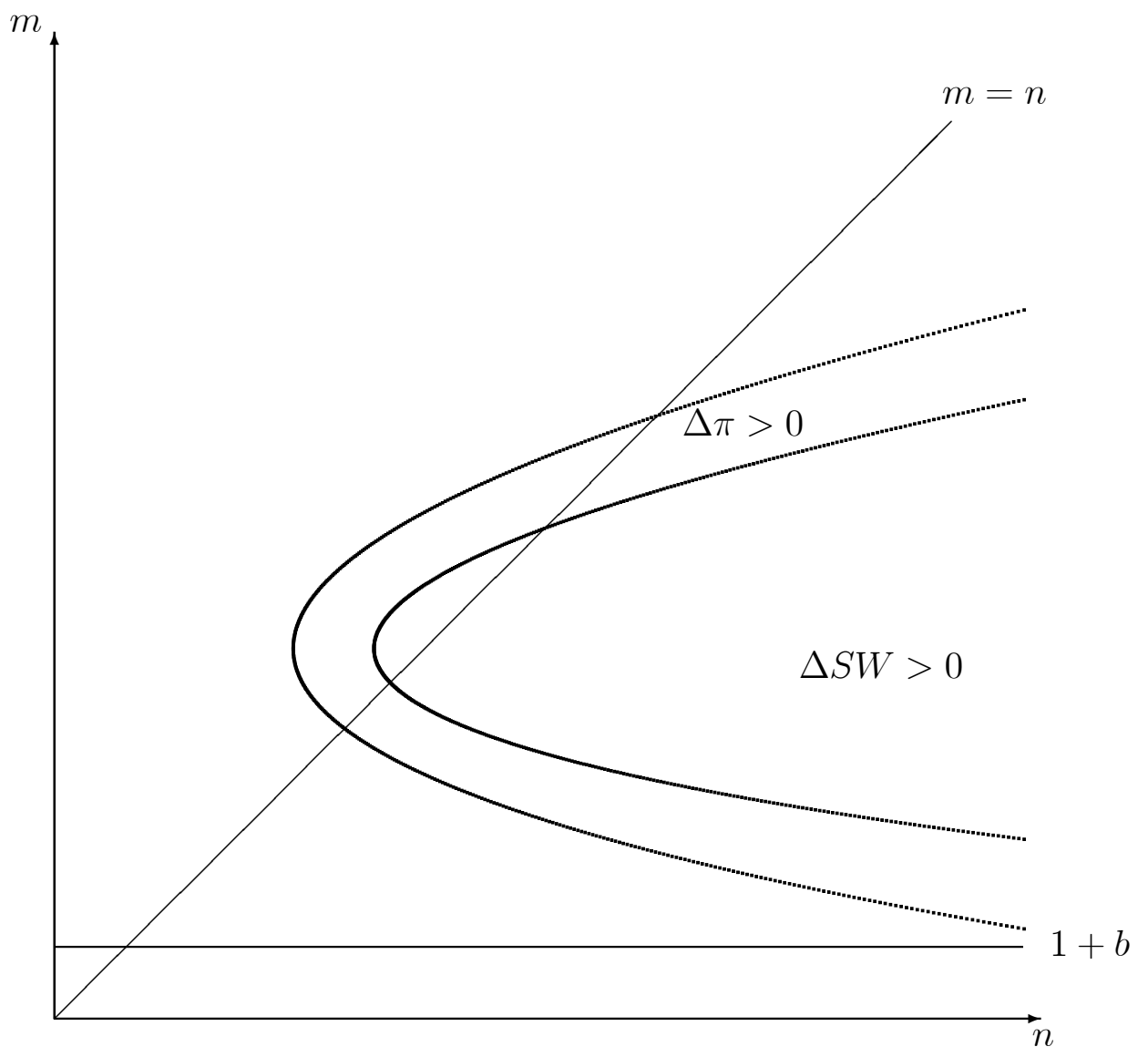


Figure 2

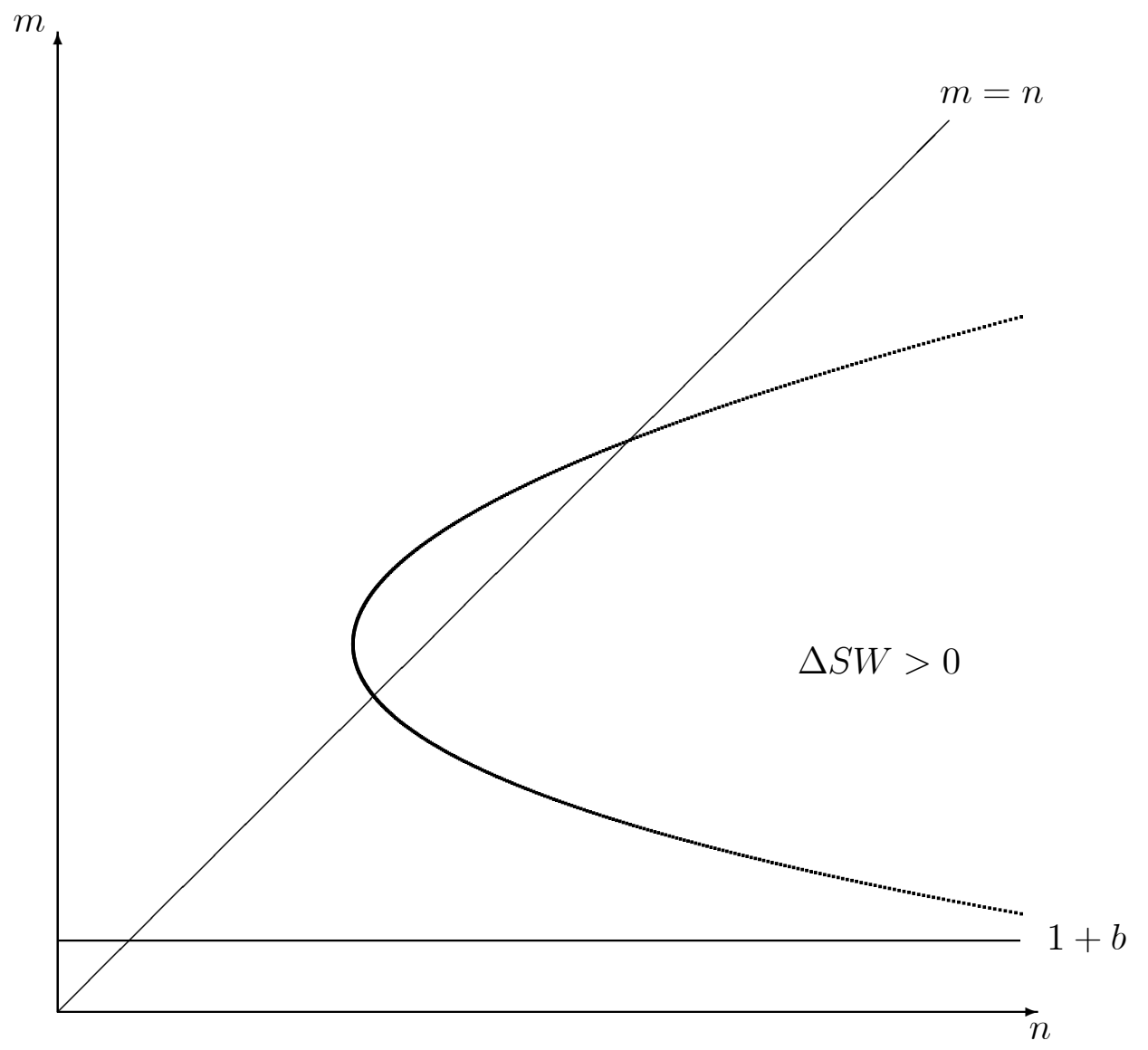




\section{Figure 3}

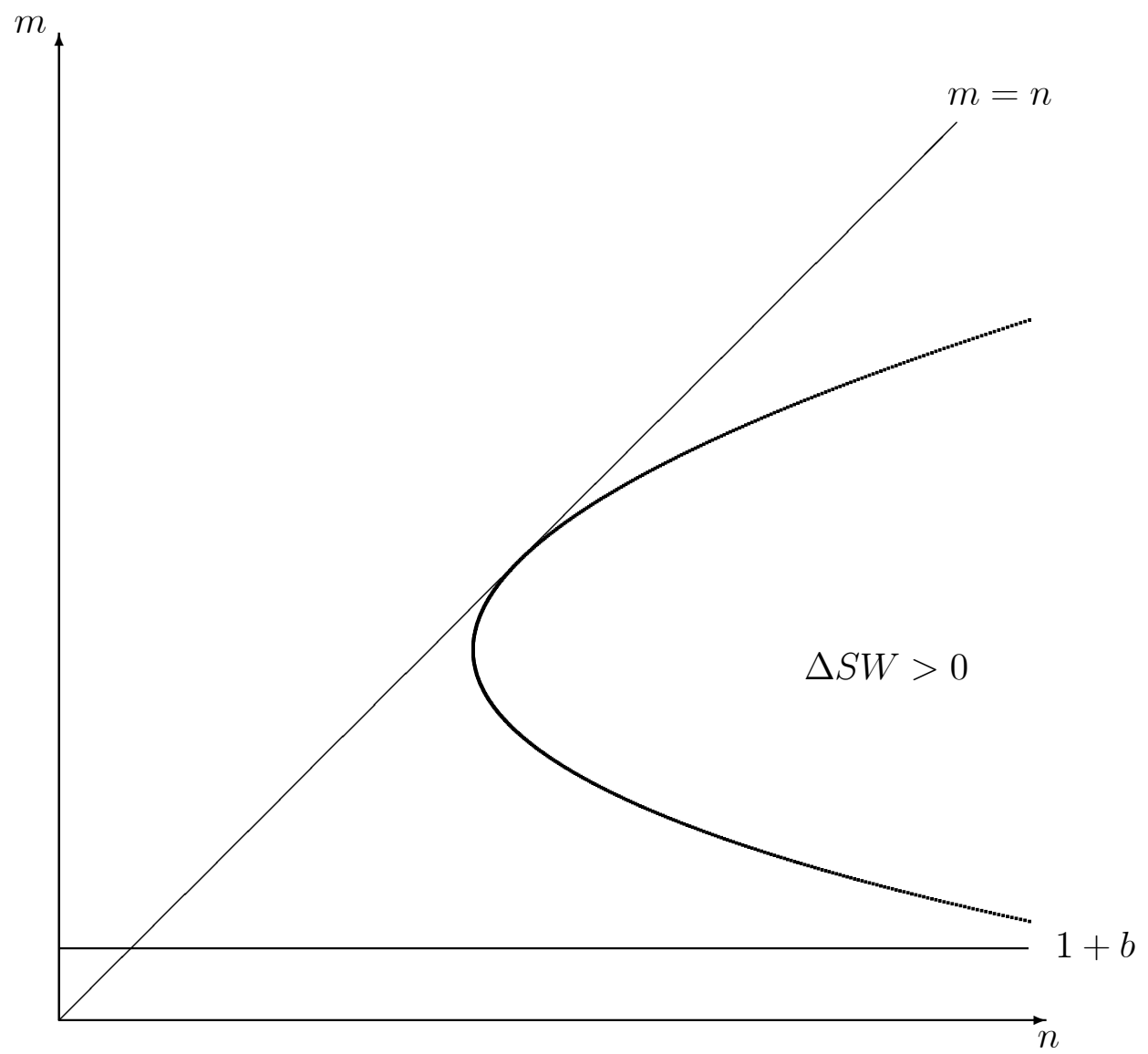

Now we turn to the comparative assessment of the results emerging from our analysis and the FTC guidelines, using a similar approach. Figure 4 plots the FTC rules against the locus $\Delta S W=0$, for values of $\left(b, F, q_{e a}\right)$ such that $\Delta S W=0$ never intersect $m=n$. Note that the condition $\Delta H H I<100$ corresponds to the triangle indicated by the arrow, while the area where $\triangle H H I<50$ is never relevant as it lies further to the right and always below 
the line $H H I=1800$.

\section{Figure 4}

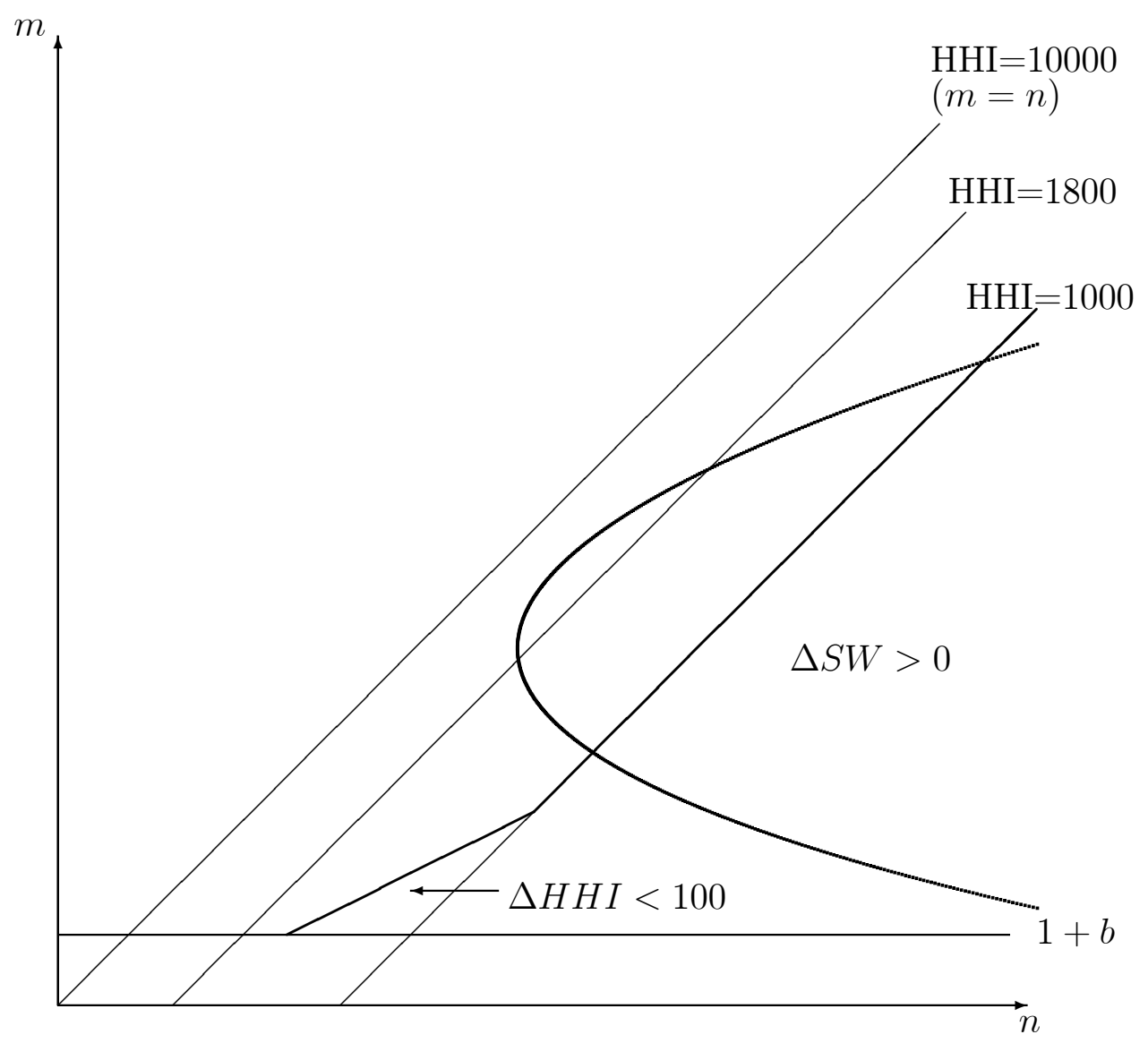

Now, the prescription produced by our model is that the antitrust agency should permit the merger in the convex area inside the locus $\Delta S W=0$. Yet, the FTC merger guidelines allow for the merger to take place in the area below and to the right of the envelope of $\triangle H H I=100$ and $H H I=1000$. Clearly, the two rules have a region in common, where the economic model suggests that the merger is socially convenient and indeed the FTC guidelines 
point in the same direction. Similarly, there's a region where the model suggests not to carry out the merger and indeed the FTC guidelines do not allow it. However, there are subsets of the parameter space where FTC rules are in conflict with the prescriptions of the economic model and produce mistakes of both types. For instance, if we take the region above the envelope of $\triangle H H I=100$ and $H H I=1000$, and inside the locus $\Delta S W=0$, we observe that here the merger is socially convenient but it is not allowed according to the FTC guidelines. Exactly the opposite happens in the region below the envelope of $\triangle H H I=100$ and $H H I=1000$, and outside the locus $\Delta S W=0$. Here the merger is socially harmful and yet it is allowed if FTC guidelines are abide by strictly.

\section{Extensions}

Here we briefly discuss some possible extensions of the method proposed above, in three directions:

- The first deals with the case of a linear demand and linear variable costs - as in the previous case - with firms differing as to their respective marginal costs, so that the total cost function of firm $i$ is $C_{i}=c_{i} q_{i}+F$. This is a trivial extension of the basic case, where ex ante equilibrium outputs are defined in terms of all firms' marginal costs but essentially the ex post performance of the merger can be easily assessed in therms of ex ante production.

- The second perspective entails the combination of a linear market demand with a cost function of the type $C_{i}=c q_{i}^{2}+F$. In such a case, ex 
ante equilibrium outputs and price are

$$
q_{e a}=\frac{a}{2 c+n+1} ; p_{e a}=a-n q_{e a} .
$$

Therefore, solving the above system one obtains

$$
a=p_{e a}-n q_{e a} ; c=\frac{p_{e a}-q_{e a}}{2 q_{e a}}
$$

which can be used to write all ex post relevant expressions in terms of ex ante observable variables.

- The third possible extension consists in an oligopoly market where demand is hyperbolic and marginal cost is increasing:

$$
p=\frac{a}{Q} ; C_{i}=c q_{i}^{2}+F
$$

In such a case, the ex ante equilibrium is given by

$$
q_{e a}=\frac{1}{n} \sqrt{\frac{a(n-1)}{2 c}} ; p_{e a}=a \sqrt{\frac{2 c}{a(n-1)}}
$$

which entail

$$
a=n p_{e a} q_{e a} ; c=\frac{p_{e a}(n-1)}{2 n q_{e a}}
$$

\section{Concluding remarks}

We have revisited the issue of horizontal mergers in a Cournot oligopoly, under the assumption that a merger entails some efficiency gain in the form of a reduction in the overall fixed costs borne by the insider firms, i.e., those taking part into the merger itself. We have identified the threshold levels of fixed costs above which the merger is (i) privately and (ii) socially beneficial, 
and we also have outlined an elementary procedure to correctly forecast the firms' performance as well as the associated welfare level after the merger has taken place. This method can be easily used by an antitrust agency to assess the admissibility of any merger on purely economic grounds, without referring to indirect indicators such as concentration indices. To this regard, we have shown that the use of the Herfindal-Hirschman index, as currently prescribed by FTC guidelines, is indeed bound to produce systematic mistakes in some relevant regions of the parameter space. As a last step, we have also provided some suggestions as to the general applicability of our method. 


\section{References}

[1] Deneckere, R. and C. Davidson (1985), "Incentives to Form Coalitions with Bertrand Competition", RAND Journal of Economics, 16, 473-86.

[2] Farrell, J. and C. Shapiro (1990), "Horizontal Mergers: An Equilibrium Analysis", American Economic Review, 80, 107-26.

[3] Farrell, J. and C. Shapiro (2000), "Scale Economies and Synergies in Horizontal Merger Analysis", University of California at Berkeley, Economics Working Paper E00-291.

[4] Federal Trade Commission (1992), Horizontal Merger Guidelines, FTC, Washington DC.

[5] Hennessy, D. (2000), "Cournot Oligopoly Conditions under Which Any Horizontal Merger Is Profitable", Review of Industrial Organization, 17, 277-84.

[6] McAfee, R.P. and M.A. Williams (1992), "Horizontal Mergers and Antitrust Policy", Journal of Industrial Economics, 40, 181-87.

[7] Perry, M.K. and R.H. Porter (1985), "Oligopoly and the Incentive for Horizontal Merger", American Economic Review, 75, 219-27.

[8] Salant, S.W., S. Switzer and R.J. Reynolds (1983), "Losses from Horizontal Merger: The Effects of an Exogenous Change in Industry Structure on Cournot-Nash Equilibrium", Quarterly Journal of Economics, 98, 185213. 